effect of statins on HbA1c levels and to determine which statin exerts most of the effect.

Methods This was a subgroup analysis of a retrospective chart review study including patients who were admitted to King Abdulaziz Cardiac Center, National Guard Hospital in Riyadh, KSA, during January 2015 until September 2017. A total of 500 cases represent the sample size of the study. The inclusion criteria included adults older than 18 years who used either rosuvastatin or atorvastatin, while the exclusion criteria included patients on simvastatin, and patients who had a contraindication for high-intensity statins or who cannot tolerate them.

Results Our results showed the opposite to previous studies. The use of statins was not associated with a significant increase in HbA1c levels in patients with diabetes $(p=0.870)$. This could be because of good glycemic control in these patients because they had already been diagnosed as diabetic patients and were on antidiabetic medications. The impact of statins on HbA1c level in non-diabetic patients demonstrated an increased level of HbA1c $(p=0.004)$. We identified which type of statin and which dose exerted most of the effect. Atorvastatin $80 \mathrm{mg}$ and rosuvastatin $20 \mathrm{mg}$ were associated with a higher rate of HbA1c disturbances in patients with diabetes only $(p=0.000$ and $\mathrm{p}=0.022$, respectively).

Conclusion This study along with previous studies showed that statins significantly affect the glycemic profile in non-diabetic patients only and showed no effect in diabetic population. The effect was significant only with high intensity statins. Therefore, careful monitoring in non-diabetic statin users is warranted.

\section{RENAL OUTCOME IN PATIENTS WHO UNDERWENT CARDIAC SURGERY USING CARDIOPULMONARY BYPASS}

Mohammed Saad Al Qarni, Abdulkarim Walid Abukhodair, Abdullah Ghunaim, Sean Russel Bennett, Andreas Fernandez Mosterio. King Faisal Cardiac Center National Guard Hospital

\subsection{6/bmjoq-2019-PSF.43}

Background Renal dysfunction is a significant variable in determining the outcome of the surgeries used to treat cardiovascular diseases - the most common being cardiopulmonary bypass graft (CABG) and valvular replacement (VR). In Saudi Arabia, the incidence of renal failure and diabetes is higher than that in most western populations. Our aim is to determine the renal outcome of patients who underwent cardiac surgery at King Faisal Cardiac Center (KFCC) over the past 3 years and explore the risk of preoperative renal function on the outcome.

Methods We conducted a retrospective cohort study of all patients older than 18 years who underwent cardiac surgery at KFCC from May 2014 to June 2017. Data were analyzed using the statistical package IBM SPSS 22.

Results The 244 patients who underwent cardiac surgery in this study period had a mean age of $60.5 \pm 7.5$ years and mean body- mass index (BMI) of $28.62 \pm 5.19$. The patients in this sample had median postoperative creatinine of 77 (IQR 66-99.75) and median bypass time of 117 (IQR 84143). There were five (2\%) patients who died within 30 days, ten (4\%) patients with temporary dialysis, 19 (7.8\%) patients with postoperative renal dysfunction, and no patients with permanent dialysis. The data showed a significant relationship between levels of preoperative creatinine and postoperative renal dysfunction $(\mathrm{p}=0.0001$, odds ratio $1.05,95 \%$ CI 1.031-1.064). Time on bypass $(p=0.998)$, hypertension $(p=1.969)$, and diabetes $(p=1.061)$ were all not significant. CABG was done in $180(72 \%)$ of the patients.

Conclusion The main predictor for poor renal outcomes for cardiac surgery is preoperative creatinine, while other variables such as age, sex, BMI, cardiopulmonary bypass time, diabetes, hypertension, and dyslipidemia did not show any risk to the renal outcome.

\section{ASSESSMENT OF THE KNOWLEDGE OF INSERTING AND MAINTAINING CENTRAL LINES IN THE ADULT INTENSIVE CARE UNITS AT KING ABDULAZIZ MEDICAL CITY - RIYADH}

Farah Khalid Alhamidy, Hanan H Balkhy, Rasha Suleiman Almahamoud, Walaa Mowaffag Alanazi, Maha Abdullah Alfarhan. College of Medicine, King Saud bin Abdulaziz University for Health Sciences

\subsection{6/bmjoq-2019-PSF.44}

Background Central line-associated bloodstream infections (CLABSIs) create a huge burden of cost on healthcare organizations. Reducing the incidence of CLABSI will not only provide better healthcare and safety to patients but will also reduce the costs associated with additional days of stay. Central venous catheters are used in the healthcare system for a variety of indications, and bloodstream infections are one of the major complications. Central line bundles are a group of guidelines created to minimize and prevent infections acquired during administration. The purpose of our research was to assess the knowledge of central line bundles among healthcare workers in intensive care units.

Methods This cross-sectional study was done in King Abdulaziz Medical City in Riyadh and included 171 nurses and 41 physicians working in the three intensive care units (surgical, medical, cardiac). Participants' awareness of central line bundles was assessed using a questionnaire that included three sections: demographics, knowledge, and practice.

Results The vast majority of the healthcare workers answered questions correctly regarding knowledge of CLABSI prevention (wearing maximal barrier precautions, washing hands, using chlorhexidine at insertion site, documenting the procedures, etc). The average knowledge score among participants was $82 \%$. A major factor determining knowledge of central line bundles is training. $60 \%$ of the participants had received central line training. Participants who received CLABSI bundle achieved a high mean score of $84 \%$, whereas this score was lower in those who had not received training (78\%).

Conclusion Our results suggest that receiving CLABSI training is associated with better knowledge and lower complications rate. Educational interventions should be used to address the gaps regarding knowledge and practice regarding the prevention of CLABSI and to ensure that healthcare workers use evidence-based prevention interventions. 